\title{
CGT Method of Message forwarding
}

\author{
Kavya.S. ${ }^{1}$, Karthika.K. ${ }^{2}$, Lakshmi Priya.G. ${ }^{3}$, Mary Varsha Peter ${ }^{4}$, Priya.M. ${ }^{5}$, Christo Ananth ${ }^{6}$ \\ U.G. Scholars, Department of ECE, Francis Xavier Engineering College, Tirunelveli ${ }^{1,2,3,4,5}$ \\ Associate Professor, Department of ECE, Francis Xavier Engineering College, Tirunelveli ${ }^{6}$
}

\begin{abstract}
In vehicular ad hoc networks (VANETs), because of the nonexistence of end-to-end connections, it is essential that nodes take advantage of connection opportunities to forward messages to make end-to-end messaging possible. Thus, it is crucial to make sure that nodes have incentives to forward messages for others, despite the fact that the routing protocols in VANETs are different from traditional end-to-end routing protocols. In this paper, stimulation of message forwarding in VANETs is concerned. This approach is based on coalitional game theory, particularly, an incentive scheme for VANETs is proposed and with this scheme, following the routing protocol is in the best interest of each node. In addition, a lightweight approach is proposed for taking the limited storage space of each node into consideration.
\end{abstract}

Index Terms - Coalitional game, cooperation, game theory, VANET

\section{INTRODUCTION}

The primary VANET's goal is to increase road safety. To achieve this, the vehicles act as sensors and exchange warnings or - more generally - telematics information (like current speed, location or ESP activity) that enables the drivers to react early to abnormal and potentially dangerous situations like accidents, traffic jams or glaze. The information provided by other vehicles and stationary infrastructure might also be used for driver assistant systems like adaptive cruise control (ACC) or breaking assistants. In addition, authorized entities like police or firefighters should be able to send alarm signals and instructions.

Mostly interests to MANETS belong to the VANETS but the features are different. Vehicles are likely to move in structured way. The connection with wayside equipment can similarly be indicated absolutely accurately. In the end, mostly automobiles are limited in their motion range, such as being controlled to pursue a paved way.

VANET suggests unlimited advantage to companies of any size. Vehicles access of fast speed internet which will change the automobiles' on-board system from an effective widget to necessary productivity equipment, making nearly any internet technology accessible in the car. Thus this network does pretend specific security concerns as one problem is no one can type an email during driving safely.
This is not a potential limit of VANET as productivity equipment. It permits the time which has wasted for something in waiting called "dead time", has turned into the time which is used to achieve tasks called "live time".

If a traveler downloads his email, he can transform jam traffic into a productive task and read on-board system and read it himself if traffic stuck. One can browse the internet when someone is waiting in car for a relative or friend. If GPS system is integrated it can give us a benefit about traffic related to reports to support the fastest way to work. Finally, it would permit for free, like Skype or Google Talk services within workers, reducing telecommunications charges.

Safety applications would be Slow/Stop Vehicle Advisor (SVA) in which a slow or stationary vehicle will broadcast warning message to its neighbourhood. Another similar type of application is emergency electronic brake-light (EEBL). In Post Crash Notification (PCN), a vehicle involved in an accident would broadcast warning messages about its position to trailing vehicles so that it can take decision with time in hand as well as to the highway patrol for tow away support. Road Hazard Control Notification (RHCN) deals with cars notifying other cars about road having landslide. Another related application would be road feature notification which deals with notification due to road curve, sudden downhill etc. Cooperative Collision Warning (CCW) alerts two drivers potentially under crash route so that they can mend their ways.

Congested Road Notification (CRN) detects and notifies about road congestions which can be used for route and trip planning. TOLL is yet another application for vehicle toll collection at the toll booths without stopping the vehicles. Parking Availability Notification (PAN) helps to find the availability of slots in parking lots in a certain geographical area.

Remote Vehicle Personalization / Diagnostics (RVP/D) helps in downloading of personalized vehicle settings or uploading of vehicle diagnostics from/to infrastructure. Service Announcements (SA) would be of particular interest to roadside business like petrol pumps, highways restaurants to announce their services to the drivers within communication range. Content Map Database Download (CMDD) acts as a portal for getting valuable information from mobile hotspots or home stations. Using Real Time Video Relay (RTVR), on-demand movie experience will not be confined to the constraints of the home and the driver can ask for real time video relay of his favorite movies.

The inter-vehicle communication configuration uses multi-hop multicast/broadcast to transmit traffic related 
International Journal of Advanced Research in Management, Architecture, Technology and Engineering (IJARMATE)

information over multiple hops to a group of receivers. There are two types of message forwarding in inter-vehicle communications i)Naïve broadcasting ii)Intelligent broadcasting.

In naïve broadcasting, vehicles send broadcast messages periodically and at regular intervals.Upon receipt of the message, the vehicle ignores the message if it has come from a vehicle behind it. If the message comes from a vehicle in front, the receiving vehicle sends its own broadcast message to vehicles behind it. This ensures that all enabled vehicles moving in the forward direction get all broadcast messages. The limitations of the naïve broadcasting method is that large numbers of broadcast messages are generated, therefore, increasing the risk of message collision resulting in lower message delivery rates and increased delivery times.

In intelligent broadcasting addresses the problems inherent in naïve broadcasting by limiting the number of messages broadcast for a given emergency event. If the event-detecting vehicle receives the same message from behind, it assumes that at least one vehicle in the back has received it and ceases broadcasting. The assumption is that the vehicle in the back will be responsible for moving the message along to the rest of the vehicles. If a vehicle receives a message from more than one source it will act on the first message.

The vehicle-to-roadside communication represents a single hop broadcast where the roadside unit sends a broadcast message to all equipped vehicles in the vicinity. Vehicle-to-roadside communication configuration provides a high bandwidth link between vehicles and roadside units. The roadside units may be placed every kilometer or less, enabling high data rates to be maintained in heavy traffic.

For instance, when broadcasting dynamic speed limits, the roadside unit will determine the appropriate speed limit according to its internal timetable and traffic conditions. The roadside unit will periodically broadcast a message containing the speed limit and will compare any geographic or directional limits with vehicle data to determine if a speed limit warning applies to any of the vehicles in the vicinity. If a vehicle violates the desired speed limit, a broadcast will be delivered to the vehicle in the form of an auditory or visual warning, requesting that the driver reduce his speed. The routing-based communication is a multi-hop unicast where a message is propagated in a multi-hop fashion until the vehicle carrying the desired data is reached. When the query is received by a vehicle owning the desired piece of information, the application at that vehicle immediately sends a unicast message containing the information to the vehicle it received the request from, which is then charged with the task of forwarding it towards the query source.

Routing protocols such as RIP and EIGRP are Distance Vector routing protocols. These are called Distance Vector protocols because they base routing decisions on the "distance" of the remote destination in terms of the number of network layer hops that the packet will have to traverse.OSPF and IS-IS are Link State routing protocols. They are called Link State protocols because they base routing decisions on messages received from other routers in the internetwork that give information about the state of the links connected to them.

Game theory is the formal study of conflict and cooperation. Game theoretic concepts apply whenever the actions of several agents are interdependent. These agents may be individuals, groups, firms, or any combination of these. The concepts of game theory provide a language to formulate, structure, analyze, and understand strategic scenarios.

The object of study in game theory is the game, which is a formal model of an interactive situation. It typically involves several players; a game with only one player is usually called a decision problem. The formal definition lays out the players, their preferences, their information, the strategic actions available to them, and how these influence the outcome.

A coalitional (or cooperative) game is a high-level description, specifying only what payoffs each potential group, or coalition, can obtain by the cooperation of its members. What is not made explicit is the process by which the coalition forms. Cooperative game theory investigates such coalitional games with respect to the relative amounts of power held by various players, or how a successful coalition should divide its proceeds.

Ad hoc networks rely on the cooperation of participating nodes to route data between source and destination pairs that are outside each other's communication range. Because such data forwarding consumes valuable (and scarce) battery power, each node along the path has an inherent disincentive to cooperate. This tension between cooperation and cost invites a game-theoretic study, where each node must strategically decide the degree to which it must volunteer its resources for the common good of the network.

A game is cooperative if the players are able to form binding commitments. For instance the legal system requires them to adhere to their promises. In noncooperative games this is not possible. communication among players is allowed in cooperative games, but not in noncooperative ones. Noncooperative games are able to model situations to the finest details, producing accurate results. Cooperative games focus on the game at large.. Hybrid games contain cooperative and non-cooperative elements. For instance, coalitions of players are formed in a cooperative game, but these play in a non-cooperative fashion.

A symmetric game is a game where the payoffs for playing a particular strategy depend only on the other strategies employed, not on who is playing them. If the identities of the players can be changed without changing the payoff to the strategies, then a game is symmetric. Asymmetric games are games where there are not identical strategy sets for both players. For instance, the ultimatum game and similarly the dictator game have different strategies for each player. It is possible, however, for a game to have identical strategies for both players, yet be asymmetric. For example, the game pictured to the right is asymmetric despite having identical strategy sets for both players. 
International Journal of Advanced Research in Management, Architecture, Technology and Engineering (IJARMATE)

Vol. 1, Issue 1, August 2015

Zero-sum games are a special case of constant-sum games, in which choices by players can neither increase nor decrease the available resources. In zero-sum games the total benefit to all players in the game, for every combination of strategies, always adds to zero. Many games studied by game theorists (including the famous prisoner's dilemma) are non-zero-sum games, because some outcomes have net results greater or less than zero. Informally, in non-zero-sum games, a gain by one player does not necessarily correspond with a loss by another.

Simultaneous games are games where both players move simultaneously, or if they do not move simultaneously, the later players are unaware of the earlier players' actions (making them effectively simultaneous). Sequential games (or dynamic games) are games where later players have some knowledge about earlier actions. This need not be perfect information about every action of earlier players; it might be very little knowledge. For instance, a player may know that an earlier player did not perform one particular action, while he does not know which of the other available actions the first player actually performed.

The difference between simultaneous and sequential games is captured in the different representations .Often, normal form is used to represent simultaneous games, and extensive form is used to represent sequential ones. The transformation of extensive to normal form is one way, meaning that multiple extensive form games correspond to the same normal form. Consequently, notions of equilibrium for simultaneous games are insufficient for reasoning about sequential games.

\section{EXISTING SYSTEM}

Vehicular ad hoc networks (VANETs)[1] are envisioned to provide promising applications and services. One critical deployment issue in VANETs is to motivate vehicles and their drivers to cooperate and contribute to packet forwarding in vehicle-to-vehicle or vehicle-to-roadside communication. To achieve fairness and provide motivative stimulation for participation, a Fair Reimbursement And Motivating sweepstake scheme (FRAME) is proposed. The FRAME scheme contains two major components: a weighted rewarding component and a sweepstake component. Based on the contribution measurement, the weighted rewarding component utilizes a convex function to calculate each vehicle's weight and allocate rewards according to the calculated weights. The sweepstake component awards a fixed amount to the winning vehicle that participates in the forwarding. The FRAME scheme is used to overcome the drawbacks of two straight forward schemes such as Receipt counting method and Proportional rewarding method.

A variable-rate and variable-power MQAM modulation scheme [2] is proposed for high-speed data transmission over fading channels. The spectral efficiency of our variable-rate variable-power MQAM technique is determined and compare with the Shannon capacity limit, where capacity is achieved using adaptive transmission techniques. High speed wireless data transmission requires robust and spectrally efficient communication techniques for flat-fading channels. When the channel can be estimated and this estimate sent back to the transmitter, the transmission scheme can be adapted relative to the channel characteristics. The variable-rate variable-power techniques increase the spectral efficiency of a single channel. This technique suffers a large power penalty since most of the average signal power is used to compensate for deep fades. It will increase the co channel interference. The aim of [3] is to construct a class of local bounds for the $\mathrm{EP}$, which improves the generic bounds in a given region. The behavior of error probability (EP) as a function of signal-to-noise ratio (SNR) and other system parameters is fundamental for assessing the design of digital wireless communication systems. An analytical framework based on the log-concavity property of the EP is proposed. To prove this property for a class of signals with constellations on a multidimensional regular grid in the presence of Gaussian distributed disturbances such as thermal noise and interference. The behavior of the EP is important for local bounds. the EP is reported on log-scale and investigated as a function of the signal-to-total disturbance ratio in decibel $(\mathrm{dB})$. The advantage is, it uses tighter bounds in the region of interest. But it increase in complexity with the usage of log-concavity property.

The aim of [4] is to provide routing support for ad-hoc, wireless networks under conditions of sporadic connectivity and ever-present network partitions. Then the proposed algorithms that exploit the relative position of stationary devices and non-randomness in the movement of mobile agents in the network. In order to pass packets between these stationary isolated agents, packets can be transmitted to passing mobile agents: wearable computers, palmtops, or laptops carried by a human, robot, mail cart, or elevator. From there, the packet might make several hops between mobile and stationary agents as it makes its way to its destination. The main tradeoff is the additional storage at nodes as packets are stored, carried, and forwarded to the destination. Epidemic routing is designed to maximize exposure by distributing packets as widely as possible, while requiring buffers of infinite length. We proposed a variety of drop strategies such as ,

- Drop-Random (DRA): the packet to be dropped is chosen at random.

- Drop-Least-Encountered (DLE): the packet is dropped based on the estimated likelihood of delivery.

The schemes mentioned in [5] are used to route packets efficiently through a highly partitioned network.when agents are restricted to local communication, the problem will occured. The main objectives are the development of cooperative driver assistance systems on the one hand and the development of a selforganising ad-hoc radio network as a communication. To achieve a suitable communication system, algorithms for radio ad-hoc networks with extremely high dynamic network topologies are developed and prototypes tested in the vehicles.CarTALK 2000 tries to build application clusters in three ways such as, information and warning functions, communication-based longitudinal 
International Journal of Advanced Research in Management, Architecture, Technology and Engineering (IJARMATE)

Vol. 1, Issue 1, August 2015

control systems and co-operative assistance systems. The benefits consist of savings of time costs, vehicle operating costs, accident costs and environmental costs (pollution, $\mathrm{CO} 2$ ). To determine a benefit-cost-ratio the benefits have to be related with the system costs. The effects for the individual user can be investigated by break-even-analyses that confront the benefits for the individual user with his costs .

There are two types of existing incentive mechanisms for stimulating cooperation in wireless networks:

1) Reputation-based approaches

2) Credit-based approaches

Reputation-based approaches rely on observing the behavior of neighbor nodes and punishing the detected uncooperative nodes to stimulate cooperation. In VANETs, however, for a distributed reputation system, the deviating behaviors of a selfish node are more difficult to be observed and determined by other nodes because the connections with the same nodes are occasional.

Credit-based mechanisms, to encourage cooperation by rewarding credits to the cooperative nodes. This idea is particularly appropriate for many applications in VANETs, such as disseminating advertising using vehicles. In existing works for traditional multihop networks, the credit based mechanisms depend on end-to-end connections to determine how many credits each node should receive. In VANETs since end-to-end paths are not guaranteed at all, existing credit based mechanisms cannot be used either.

\section{PROPOSED SYSTEM}

The overall architecture of the system consists of a number of smart vehicles that have VANET communication devices installed and a central authority called the virtual credit center (VCC). As in many other incentive schemes for wireless networks and, particularly, the VANET, the VCC is used. The VCC issues a certificate to each node, and each node has an account (of virtual currency) in the VCC. Nodes do not need to connect to the VCC all the time. Instead, nodes save and store the information that they need to communicate with the VCC temporarily, and when they are close to some infrastructures, they connect to the VCC and communicate with it (including receiving credits).

The complete incentive scheme consists of the programs installed at each node in the VANETs and the algorithm running at the VCC. The programs at each node can further be divided into three groups of functions:

1) The source node

2) The intermediate node

3) The destination node
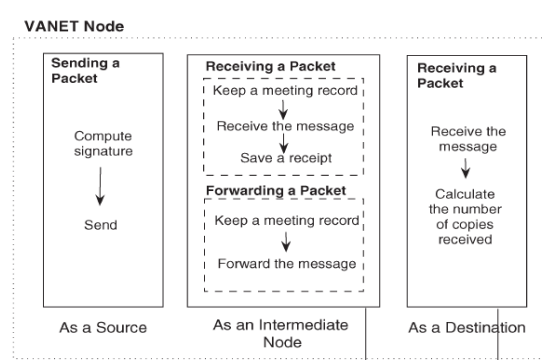

vcc

Fig.1. Incentive scheme implementation architecture

A. Hardware Requirements:

$\begin{array}{lll}\text { Processor } & : & \text { Intel Pentium IV } \\ \text { Processor Speed } & : & 1.4 \mathrm{GHz} \\ \text { Memory (RAM) } & : & 512 \mathrm{MB} \\ \text { Hard disk } & : & 40 \mathrm{~GB} \\ \text { Monitor } & : & 14 \\ \text { Input Device } & : & \text { Keyboard (104) }\end{array}$

B. Software Requirements:

Operating System : LINUX

Simulator Tool : NS2

Language : $\mathrm{C}++$ and TCL

Platform : Independent

\section{SYSTEM MODULES}

- Creation of Nodes

- $\quad$ Creation of Agents

- $\quad$ Coalitional game Model

- $\quad$ Allocation of Payoff

\section{RESULTS AND DISCUSSION}

Node configuration essentially consists of defining the different node characteristics before creating them. This may consist of the type of addressing structure used in the simulation,defining the network components for mobile nodes,turning on or off the trace options at Agent/R outer/MAC levels,selecting the type of adhoc routing protocol for wireless nodes or defining their energy model.

Adding a New Agent in NS2 uses C++ to be coded. Unlike conventional C++,NS2 uses TclCL and OTCL. There are $6 \mathrm{TclCL}$ classes that are needed to develop any module in NS2.

$$
\begin{array}{ll}
\text { - } & \text { Tcl } \\
\text { - } & \text { TclClass } \\
\text { - } & \text { EmbeddedTcl } \\
\text { - } & \text { TclObject } \\
\text { - } & \text { TclCommand }
\end{array}
$$

In a coalitional game, there are a number of players. These players correspond to the nodes in a VANET. When the players in a subset decide to cooperate within the subset, the subset is called a coalition. In particular, the coalition of 
International Journal of Advanced Research in Management, Architecture, Technology and Engineering (IJARMATE)

all players is called the grand coalition. Hence, whenever a message needs to be forwarded in a VANET, all involved nodes have incentives to form a grand coalition.

In coalitional game theory, the central concept is the formation of coalitions. Each coalition is a subset of game players who cooperatively join forces. Each selfish player always tries to join the coalition that can maximize its own payoff share. All players join the grand coalition so that any two players cooperate with each other. Since each player has the freedom to choose the coalition to join based on its own interests, it may ensure that joining the grand coalition is in the best interest of every player.

To achieve the grand coalition, the core in the game and payoff allocation to each player in the coalition that satisfies the core requirement.

For each intermediate node, its share of payoff should reflect its contribution in the game. Hence, the payoff-allocation function for intermediate nodes is designed as based on two types of behaviors in the coalition, i.e., receiving and forwarding.

$x_{i}=\alpha \cdot m_{r}(i)+\beta \cdot m_{f}(i)+u \cdot n_{r \in o}(i) \quad \forall i \neq s r c$

where, $m_{r}(i)$ is the number of times that the intermediate node I receives one copy of the message from some other node. $m_{f}(i)$ is the number of times that i successfully forwards one copy of the message to another node following the routing protocol $\alpha \alpha$ and $\beta$ are the rewards for the receiving and forwarding behaviors, respectively. $u . n_{r e c}(i)$ is the amount of reward to node i for reporting the meeting records. Note that dest. Can be viewed as an intermediate node, which only receives copies without further forwarding.

- Payoff Allocation to the Source Node

The payoff allocation to the source node contains two parts: the gains by successfully delivering message copies to dest. subtracted by rewards used to pay the intermediate nodes. The payoff allocation function for src. is defined as

$x_{m e}=\delta . d(N)-\left(\alpha \sum_{i \in N-\{s r o\}}^{\prime} m_{r}(i)+\beta \sum_{i \in N-\{s r o\}} m_{f}(i)\right)$

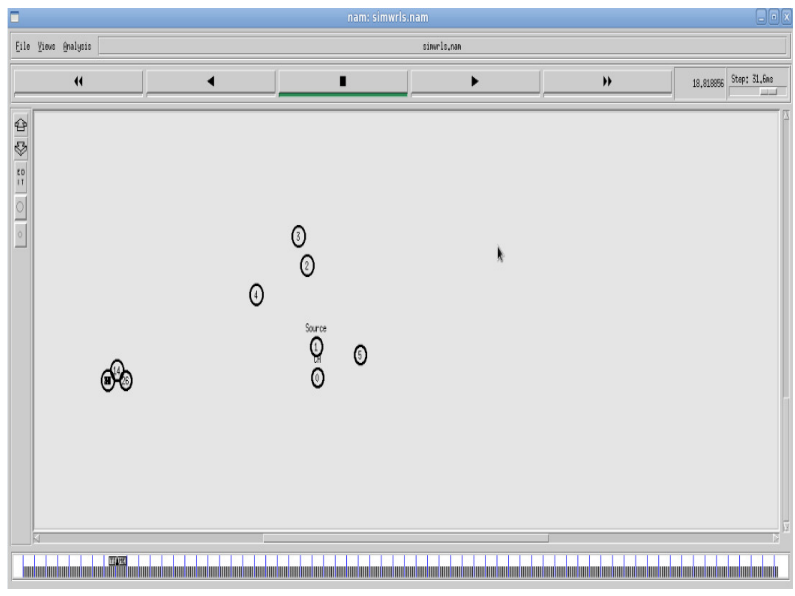

Fig.2. Moving nodes due to mobility

Since its a dynamic topology Fig.2. shows about the nodes moving due to mobility.

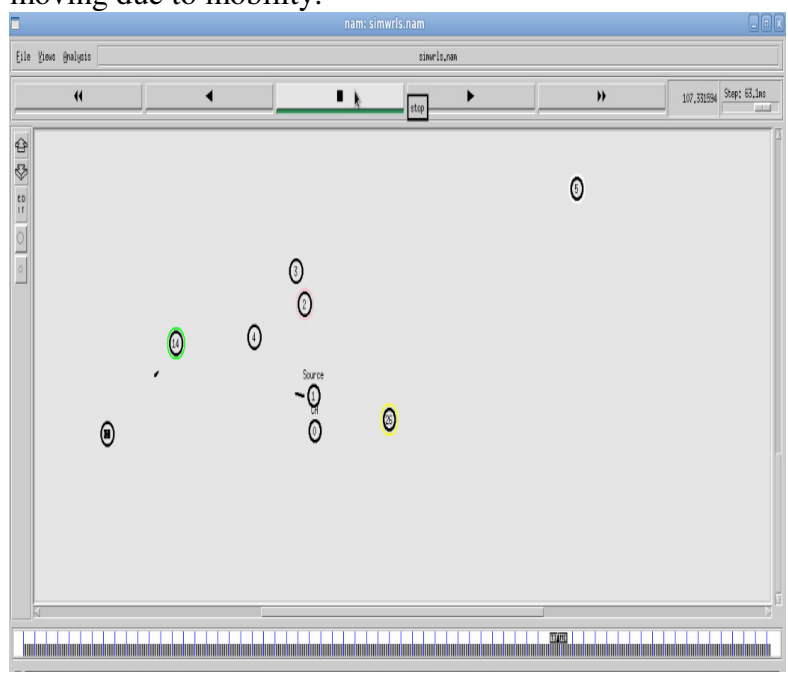

Fig.3. Message forwarding between nodes

Fig.3. shows the message forwarding between the needed mobile nodes.

\section{CONCLUSION}

In vehicular ad hoc networks (VANETs), because of the nonexistence of end-to-end connections, it is essential that nodes take advantage of connection opportunities to forward messages to make end-to-end messaging possible. Thus, it is crucial to make sure that nodes have incentives to forward messages for others, despite the fact that the routing protocols in VANETs are different from traditional end-to-end routing protocols. In this paper, stimulation of message forwarding in VANETs is concerned. This approach is based on coalitional game theory, particularly, an incentive scheme for VANETs is proposed and with this scheme, following the routing protocol is in the best interest of each node. In addition, a 
lightweight approach is proposed for taking the limited storage space of each node into consideration.

\section{REFERENCES}

[1]J. Burgess, B. Gallagher, D. Jensen, and B. N. Levine, "MaxProp: Routing for vehicle-based disruption-tolerant networks," in Proc. INFOCOM, Apr. 2006, pp. 1-11.

[2] E. Jones, L. Li, and P. Ward, "Practical routing in delay-tolerant networks," in Proc. ACM Chants Workshop, Aug. 2005, pp. 237-243.

[3]S. Jain, K. Fall, and R. Patra, "Routing in a delay tolerant network," in Proc. SIGCOMM, 2004, pp. 145-158.

[4] A. Vahdat and D. Becker, "Epidemic routing for partially connected ad hoc networks," Duke Univ., Durham, NC, Tech. Rep. CS-200006, Apr. 2000

[5]B. Burns, O. Brock, and B. N. Levine, "MV routing and capacity building in disruption tolerant networks," in Proc. INFOCOM, Mar. 2005, pp. 398-408.

[6] T. Spyropoulos, K. Psounis, and C. S. Raghavendra, "Spray and wait: An efficient routing scheme for intermittently connected mobile networks," in Proc. WDTN, Aug. 2005, pp. 252-259.

[7]S. Marti, T. Giuli, K. Lai, and M. Baker, "Mitigating routing misbehavior in mobile ad hoc networks," in Proc. MOBICOM, Boston, MA, Aug. 2000 pp. $255-265$.

[8] P. Michiardi and R. Molva, "CORE: A collaborative reputation mechanism to enforce node cooperation in mobile ad hoc networks," in Proc. CMS, Portoroz, Slovenia, Sep.2002, pp. 107-121.

[9] M. T. Refaei, V. Srivastava, L. DaSilva, and M. Eltoweissy, "A reputation based mechanism for isolating selfish nodes in ad hoc networks," in Proc. MobiQuitous, San Diego, CA, Jul. 2005, pp. 3-11.

[10] S. Zhong, J. Chen, and Y. R. Yang, "Sprite, a simple, cheat-proof, credit-based System for mobile ad-hoc networks," in Proc. INFOCOM, San Francisco, CA, Apr. 2003, pp. 1987-1997. 